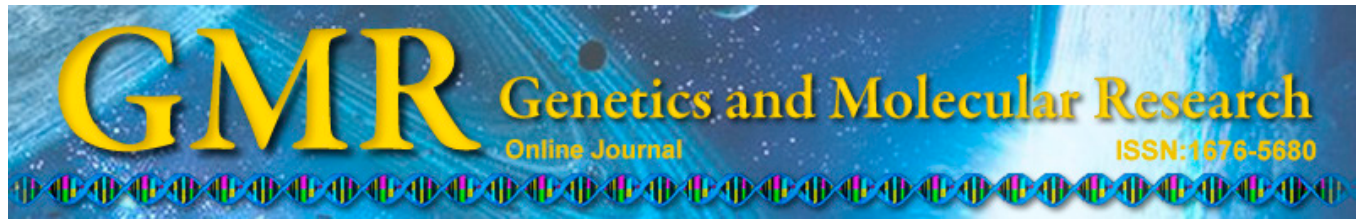

\title{
A novel method for the evaluation of virus-induced gene silencing efficiency
}

\author{
C. Li $^{1,2}$, Z.C. Zhang ${ }^{1}$, K.M. Ghebremariam ${ }^{1}$, L.H. Wang ${ }^{1}$, L. Wu ${ }^{1}$ and \\ Y. Liang ${ }^{1}$
}

${ }^{1}$ State Key Laboratory of Crop Stress Biology in Arid Region, College of Horticulture, Northwest A\&F University, Yangling, Shaanxi, China ${ }^{2}$ College of Life Science, Huaibei Normal University, Huaibei, Anhui, China

Corresponding author: Y. Liang

E-mail: liangyan@nwsuaf.edu.cn

Genet. Mol. Res. 13 (4): 9443-9452 (2014)

Received June 25, 2013

Accepted February 13, 2014

Published November 11, 2014

DOI http://dx.doi.org/10.4238/2014.November.11.9

\begin{abstract}
Virus-induced gene silencing (VIGS) is an important tool for studying gene function. However, a number of factors highly restrict the application of VIGS, such as unstable efficiency and tissue-specific silencing. We developed a novel evaluation method for improving the applicability of VIGS vectors. In this method, 4 indexes were defined and utilized to evaluate VIGS efficiency by silencing the endogenous phytoene desaturase $(P D S)$ gene with a tobacco rattle virus-based VIGS vector. To illustrate the reliability of this evaluation method, we assessed the silencing efficiency of SpPDS and SpMPK1 in Solanum pimpinellifolium. The silencing results of $S p P D S$ showed that an optical density at $600 \mathrm{~nm}$ of 2.0 was more suitable than 1.0 for VIGS in $S$. pimpinellifolium. This suggests that the proposed evaluation method is a valid technique for optimizing the VIGS system of plants. Moreover, the SpMPK1 gene was highly silenced in the 4th-9th leaves with a 50$95 \%$ reduction in transcription levels, further demonstrating that this method can be used to select highly silenced candidates for further
\end{abstract}


experiments, particularly when the target gene shows no phenotypic change after being silenced.

Key words: Evaluation method; Virus-induced gene silencing (VIGS); Silencing efficiency; Solanum pimpinellifolium

\section{INTRODUCTION}

Virus-induced gene silencing (VIGS), an approach used for post-transcriptional gene silencing (Baulcombe, 1999), is an important tool for investigating gene function and high-throughput functional genomics in plant science by exploiting plant RNA interferencemediated antiviral defense mechanism (Liu et al., 2002b; Ekengren et al., 2003; Benedito et al., 2004; Burch-Smith et al., 2004). Viral vectors carrying sequences derived from host genes can be targeted to the mRNAs of a corresponding host using a post-infiltrated process (Bartel, 2004). Tobacco rattle virus (TRV) is one of the most frequently used vectors for VIGS because of its increased capability of spreading throughout the plant as well as its relatively mild impact on plant health relative to other viruses such as tobacco mosaic virus and potato virus X (Ratcliff et al., 2001). TRV is a bipartite, single-stranded, positive-sense RNA plant virus consisting of two types, TRV1 and TRV2; TRV2 typically harbors a coat protein and a fragment from the host gene, whereas TRV1 is responsible for encoding the viral replication and movement factors and facilitates TRV2 mobility during silencing (Liu et al., 2002b). The combination of TRV1 and TRV2 results in gene silencing in plants.

As a tool for functional analysis, TRV-based VIGS has been used in a broad range of plants, including tobacco (Ratcliff et al., 2001; Jin et al., 2002; Peart et al., 2002a,b; Ahn et al., 2004; del Pozo et al., 2004; Saedler and Baldwin, 2004; Valentine et al., 2004), tomato (Liu et al., 2002a; Ekengren et al., 2003; Brigneti et al., 2004; Ryu et al., 2004; Kandoth et al., 2007; Wu et al., 2011; Hosseini Tafreshi et al., 2012; Liu et al., 2012), pepper (Chung et al., 2004), and petunia (Chen et al., 2004; Ryu et al., 2004). However, the VIGS method has some limitations, resulting in an unstable silencing efficiency, localized tissue silencing, and variable silencing efficiencies among different genera and even within the same species caused by factors such as the length of insertion, seedling age, and culture conditions. According to previous studies, a 400-bp insertion is preferable for promoting effective gene silencing and for limiting the development of damage symptoms (Wu et al., 2011). Furthermore, previous studies have shown that 7-, 14-, or 21-day-old tomato seedlings, grown at $18-21^{\circ} \mathrm{C}$ are suitable for VIGS (Liu et al., 2002a; Ekengren et al., 2003; Velásquez et al., 2009; Wu et al., 2011). Studies aimed at improving VIGS efficiency have been reported in Solanum; however, silencing is not $100 \%$ efficient. Few studies have been conducted using other plants. Thus, an evaluation method is needed for assessing VIGS efficiency to optimize and select for highly silenced candidates.

In this study, we developed a novel VIGS evaluation method. To illustrate the reliability of this method, we assessed VIGS efficiency by silencing the endogenous phytoene desaturase gene $(S p P D S)$ with a TRV-based VIGS vector in Solanum pimpinellifolium. In addition, the results were validated by successful silencing of the SPMPK1 gene in S. pimpinellifolium. Our data strongly indicate that this method is applicable for evaluating the VIGS system and highly silenced candidates. Moreover, our method can be applied for optimizing the VIGS system in other plants, thereby improving the applicability of VIGS vectors. 


\section{MATERIAL AND METHODS}

\section{Plant material and growth conditions}

S. pimpinellifolium, commonly known as the proposed wild progenitor of domesticated tomato (van der Knaap et al., 2004), was used in this study. Seeds of S. pimpinellifolium (L03708) were cultivated in cell trays filled with matrix in an artificial climate incubator at $21^{\circ} \pm 2{ }^{\circ} \mathrm{C}$ with $50 \%$ relative humidity and a 16 -h photoperiod with light intensity at $300-400 \mu \mathrm{mol} \cdot \mathrm{m}^{-2} \cdot \mathrm{s}^{-1}$.

\section{TRV vector construction and agroinfiltration in $S$. pimpinellifolium}

The pTRV1 and pTRV2 VIGS vectors described previously (Liu et al., 2002b) were obtained from Dr. S.P. Dinesh-Kumar (University of California, Davis, CA, USA). The insertion was amplified by polymerase chain reaction (PCR) using a cDNA template synthesized using the PrimeScript RT reagent Kit (Takara, Dalian, China) from total RNA extracted from leaf tissues of S. pimpinellifolium L03708 using Trizol reagent (Invitrogen, Carlsbad, CA, USA). The following primer pairs were used: 5'-GGCGCGAGCTCGGCACTCAACTTTA TAAACC-3' and 5'-CGGCGCTCGAGCTTCAGTTTTCTGTCAAACC-3' (XhoI and SacI sites are underlined). A 409-bp product of $S p P D S$ obtained from the digestion after PCR was cloned into the respective sites of pTRV2 plasmid digested with $X h o \mathrm{I} / \mathrm{SacI}$ to produce the pTRV2:PDS plasmid. A 200-bp fragment of SpMPK1, which was PCR-amplified from the cDNA template using a pair of primers (forward: 5'-GGCGCGAGCTCATAATTGCTGAC AGATTGTTGC-3' and reverse: 5'-CGGCGCTCGAGCATTTCAGTCTAAAATAAAATCC AC-3'), was linked to pTRV2 to produce the pTRV2:MPK1 plasmid.

For the VIGS assays, pTRV1, pTRV2, pTRV2:PDS, and pTRV2:MPK1 were introduced into the Agrobacterium tumefaciens strain GV3101 by electroporation. A colony was grown at $28^{\circ} \mathrm{C}$ overnight with shaking at $200 \mathrm{rpm}$ in Luria-Bertani broth containing antibiotics (50 $\mu \mathrm{g} / \mathrm{mL}$ kanamycin, $10 \mu \mathrm{g} / \mathrm{mL}$ rifampicin, and $50 \mu \mathrm{g} / \mathrm{mL}$ gentamycin). Next, secondary liquid induction media containing antibiotic and $200 \mu \mathrm{M}$ acetosyringone was used to culture the bacteria to an optical density at $600 \mathrm{~nm}\left(\mathrm{OD}_{600}\right)$ of $0.5-0.8$. Cells were harvested and resuspended twice in suspension media ( $10 \mathrm{mM} \mathrm{MgCl}_{2}, 10 \mathrm{mM}$ MES, $200 \mathrm{mM}$ acetosyringone, $\mathrm{pH}$ 5.5) (Ahn et al., 2004), and the sample was adjusted to an $\mathrm{OD}_{600}$ of 1.0 or 2.0 and incubated at room temperature for $3 \mathrm{~h}$. The mixture of pTRV1 and pTRV2:PDS/pTRV2:MPK1 in a 1:1 ratio was prepared and infiltrated into both cotyledons as described previously (Ahn et al., 2004), using pTRV1/pTRV2:00 as a negative control.

\section{Evaluation of silencing efficiency}

The evaluation method was composed of 4 indexes, as follows: 1) Silencing frequency of plants (SFP) was defined as the percentage of plants showing a silencing phenotype compared to the total number of plants infiltrated with pTRV2:PDS. We propose this as the first index in evaluating the silencing efficiency of VIGS. The following equation was used:

$\mathrm{SFP}=\frac{\text { Number of plants showing silencing phenotype (bleaching or yellowing) }}{\text { Total number of plants infiltrated }} \times 100 \% \quad$ (Equation 1$)$ 
2) Silencing efficiency of a plant (SEP) was calculated by counting the number of leaves showing photobleaching on a gene-silenced plant.

$$
\mathrm{SEP}=\frac{\text { Number of leaves showing silencing phenotype on a plant }}{\text { Total number of leaves on that plant }} \times 100 \% \quad \text { (Equation 2) }
$$

3) Silencing frequency of each leaf (SFL) was defined as the frequency of a particular leaf showing the silencing phenotype in all gene-silenced plants at 40 days post-infiltration (dpi). This index specifies the location of silencing.

$$
\mathrm{SFL}=\frac{\text { The number of a certain leaf showing silencing phenotype }}{\text { Total number of plants showing phenotype }} \times 100 \% \quad \text { (Equation 3) }
$$

4) Silencing efficiency of gene (SEG) was defined as the quantification of gene silencing. It was computed in two ways: the reduction of gene transcription levels and the ratio of bleaching areas. The following method was used for calculation.

The reduction of gene transcription levels refers to the difference between the SpPDS gene transcription levels in control plants (defined as 100\%) and those in gene-silenced plants. To quantify the abundance of SpPDS transcription in silenced and control plants, quantitative reverse transcription (RT)-PCR was performed. Total RNA was extracted from leaves, and first-strand cDNA was synthesized using the PrimeScript RT reagent Kit (Takara). Quantitative RT-PCR was performed using SYBR Premix Ex Taq II (Takara) in an iQ5 Real-Time PCR Detection System (Bio-Rad, Hercules, CA, USA). To quantify gene transcription in silenced plants, the $2^{-\Delta \Delta \mathrm{Ct}}$ method was applied. Transcription levels of $S$. pimpinellifolium elongation factor 1- $\alpha$ were internally controlled to normalize the data for $S p P D S$ and $S p M P K 1$ transcripts. The primers used in experiment were forward: 5'-ATGCCACGACCAGA

AG-3' and reverse: 5'-TGCTGTAGACAAACCACCC-3' (for $S p P D S$ ); forward: 5'-GACAGG CGTTCAGGTAAGG-3' and reverse: 5'-CCAATGGAGGGTATTCAGC-3' (for elongation factor 1- $\alpha$ ); and forward: 5'-ATCCCAGAAGGAGAATAACAG-3' and reverse: 5'-ATCAAACCTG CAACAATCTG-3' (for SpMPK1).

$$
\text { Reduction of gene transcripts }=(1-\text { relative transcripts }) \times 100 \% \quad \text { (Equation } 4)
$$

The ratio of bleaching areas was defined as the bleached areas compared to the total area of detected leaves. Quantification was accomplished by sampling and photographing 3 leaves of each plant with the camera lens perpendicular to the flat leaves. Photographs were then imported into the Adobe Photoshop CS4 software (Adobe Systems Corp., San Jose, CA, USA) to calculate the total area of the 3 leaves as well as the bleaching and yellowing areas using the Magnetic Lasso and Straw tools.

$$
\text { Ratio of bleaching areas }=\frac{\text { total areas of bleaching and yellowing }}{\text { total area of the three leaves }} \times 100 \% \quad(\text { Equation 5) }
$$

\section{Statistical analysis}

$\mathrm{R}$ 2.15.2, which is an open-source software, was used for statistical analyses. Silencing 
efficiency of plants was analyzed by univariate analysis of variance followed by a post hoc test. Gene silencing efficiency was analyzed using the Pearson product-moment correlation test.

\section{RESULTS}

To verify the applicability of the proposed evaluation method, 2 experiments were conducted. First, an Agrobacterium suspension was prepared and infiltrated into tomato seedlings, and then the silencing efficiency of the SpPDS gene was evaluated during 15-40 dpi. In addition, to validate the accuracy and reliability of the evaluation results, silencing of the SpMPK1 gene and the concurrent evaluation of its silencing efficiency was carried out.

\section{Application of the evaluation method for assessing VIGS efficiency}

\section{Silencing efficiency of SpPDS at $O D_{600}$ of 1.0}

Plants inoculated with pTRV2:PDS $\left(\mathrm{OD}_{600}=1.0\right)$ developed photobleaching symptoms at 12 dpi; experimental data were collected until 40 dpi. As shown in Figure 1A, the SFP increased rapidly from 25 to $50 \%$ from $12-15 \mathrm{dpi}$, and then gradually increased to reach a maximum of $65 \%$ at 25 dpi. Based on these results, 35 plants showing photobleaching symptoms were used to calculate the SEP (Figure 1B, box-plot). At 15 dpi, the median line was located at $40 \%$ (indicating that $40 \%$ of the total leaves were bleached on plants on average) and then reached $60 \%$ at $20 \mathrm{dpi}$.
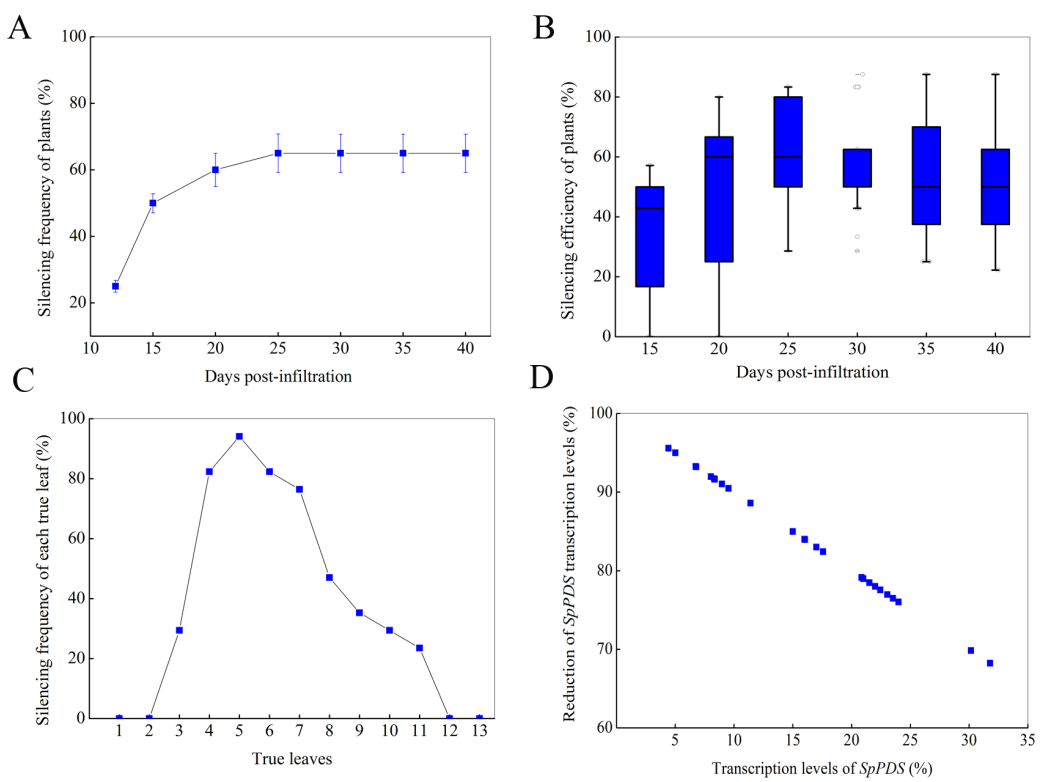

Figure 1. VIGS efficiency of $S p P D S$ at an $\mathrm{OD}_{600}$ of 1.0 in Solanum pimpinellifolium. A. Silencing frequency of plants. The values are reported as means $\pm S D$ of 3 independent experiments $(N \geq 20)$ and the bars represent the standard errors. B. Silencing efficiency of plants. Thirty-five VIGS plants were used in the experiment and the results are shown as box-plots. C. Silencing frequency of each leaf at $40 \mathrm{dpi}$. The experiments were representative of 35 VIGS plants. D. Silencing efficiency of the SpPDS gene. Leaves 4-7 from 25 VIGS plants were used in the experiment and quantitative RT-PCR was performed with 3 replicates. 
Unfortunately, long-ranging boxes were observed at 15 and $20 \mathrm{dpi}$, reflecting the instability of silencing efficiency among the plants. At 25 dpi, the SEP reached a maximum for the up-moved box, but then decreased at a later point, indicating that the newly emerged leaves did not show the silencing effect.

As described above, SFL reflects the silence-specific position within the plant. In this assay, photobleaching symptoms were observed in leaves 3-11, and SFL in leaves 4-7 reached $75-95 \%$ (Figure 1C). These results indicate that the $S p P D S$ gene was highly silenced in leaves 4-7. SEG was calculated by performing quantitative RT-PCR for leaves 4-7 from 25 VIGS plants. As shown in Figure1D, the transcription levels of the SpPDS gene ranged from 5-35\%, showing a large decrease compared to control plants. Correspondingly, SEG ranged from 65$95 \%$. These results indicate that an $\mathrm{OD}_{600}$ of 1.0 is not the optimal concentration for VIGS in S. pimpinellifolium because of the lower silencing frequency of plants, unstable silencing efficiency, and shortened duration of silencing symptoms. This suggests the successful application of the evaluation method for assessing VIGS efficiency.

\section{Correlation between the reduction of gene transcription and the ratio of bleaching areas}

The SpPDS SEG was quantified by calculating both the ratio of bleached areas and the reduction of gene transcription levels. Leaves 3-11 of VIGS plants were analyzed and the results are shown in Figure 2. In the first method, the ratio of bleaching areas ranged from $20-97 \%$. In the second method, the reduction in $S p P D S$ transcript abundance was determined using quantitative RT-PCR with RNA isolated from the same samples; transcript abundance ranged from $27-98 \%$. The results obtained using both methods showed a significant positive correlation $(\mathrm{r}=0.873, P<10 \mathrm{E}-9)$. This suggests that the reduction in $S p P D S$ gene transcription levels or the ratio of bleaching areas can be used to calculate $S p P D S$ gene silencing efficiency.

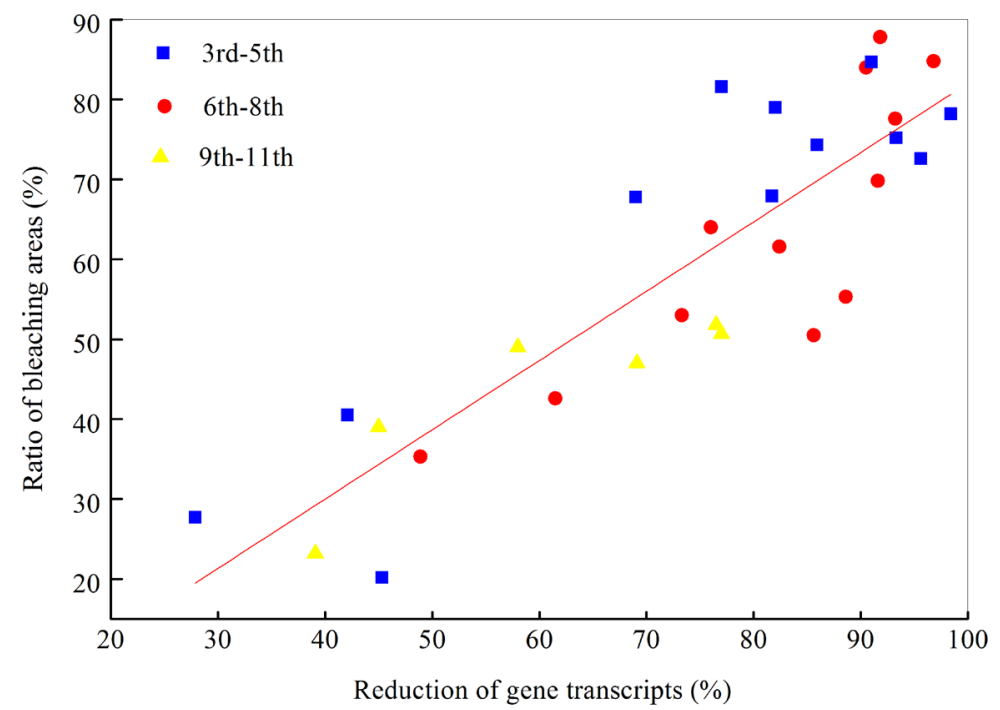

Figure 2. Correlation between the reduction of gene transcripts and the ratio of bleaching areas. Leaves 3-11 were sampled randomly from silenced plants from $15-40$ dpi $(\mathrm{r}=0.873, P<10 \mathrm{E}-9)$. 


\section{Evaluation of SpMPK1 gene silencing efficiency at $O D_{600}$ of 2.0}

To further demonstrate the general reliability of this evaluation method, we silenced the SpMPK1 gene in S. pimpinellifolium using the SpPDS gene as a positive control. Because of the lower silencing efficiency of VIGS at an $\mathrm{OD}_{600}$ of 1.0 , the infiltration concentration was increased to 2.0 in this assay.

\section{Silencing efficiency of SpPDS at $O D_{600}$ of 2.0}

Plants inoculated with an $\mathrm{OD}_{600}$ of 2.0 showed a much higher silencing frequency. Initially (12 dpi), the SFP was 50\% and then increased to $70 \%$ at 15 dpi before finally reaching a maximum (92.3\%) at 25 dpi (Figure $3 \mathrm{~A}$ and C). Thus, more than 50 VIGS plants were obtained and the SEP was calculated. As shown in Figure 3D, all box plots were relatively small throughout the experimental time, reflecting stable silencing efficiency in the plants. Median lines in box plots were located at $>50 \%$, over $70 \%$ at 25-30 dpi, and then stabilized at approximately $60 \%$ at 40 dpi. Thus, higher silencing efficiency was observed in VIGS plants.

A

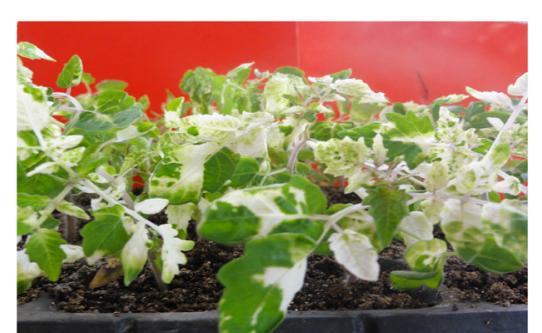

B

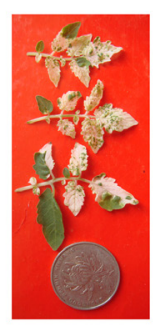

C

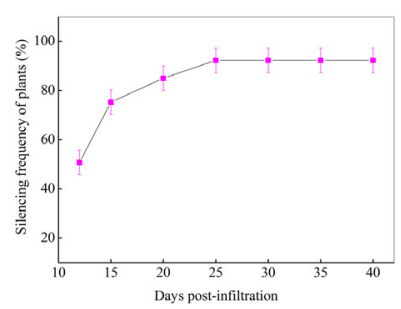

E

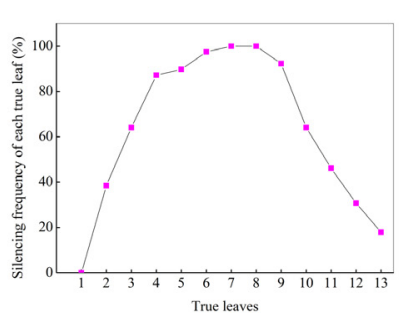

D

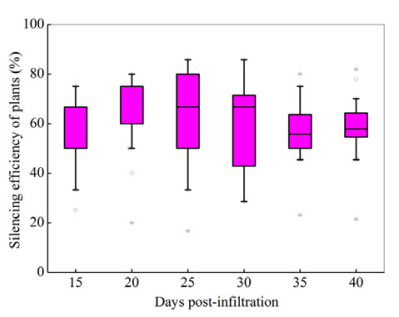

F

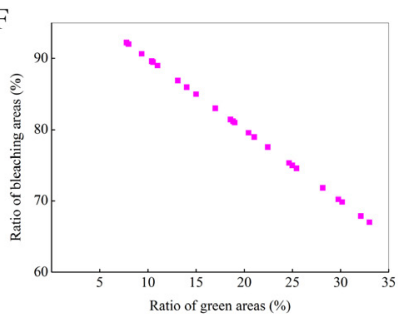

Figure 3. VIGS efficiency of $S P P D S$ at an $\mathrm{OD}_{600}$ of 2.0 in $S$. pimpinellifolium. A. Photobleaching phenotype induced by pTRV:PDS in S. pimpinellifolium. B. Leaves used to calculate the ratio of bleaching areas. C. Silencing frequency of plants. Values are reported as means \pm SD of 3 independent experiments $(N \geq 20)$ and the bars represent the standard errors. D. Silencing efficiency of plants. Fifty VIGS plants were used in the experiment; results are shown as box plots. E. Silencing frequency of each leaf at $40 \mathrm{dpi}$. The experiments were representative of 50 VIGS plants. F. Silencing efficiency of the $S p P D S$ gene. SpPDS gene silencing efficiency was assessed by the ratio of bleaching areas. The experiment was performed using leaves 4-9 from 25 VIGS plants independently. 
During the experimental time, photobleaching symptoms were observed in leaves 2-13, and the SFL of leaves 4-9 increased to $80-100 \%$ (Figure 3E). These results show that the $S p P D S$ gene may be highly silenced in this position. Concurrently, 25 VIGS plants were selected to test the SEG of leaves (4-9) by calculating the ratio of bleaching areas (Figure 3B). As predicted, the SEG was $65-95 \%$ (Figure 3F). Furthermore, more VIGS plants were obtained and more persistent photobleaching symptoms were observed when the infiltration concentration was an $\mathrm{OD}_{600}$ of 2.0 compared to an $\mathrm{OD}_{600}$ of 1.0 . These results strongly suggest that an $\mathrm{OD}_{600}$ of 2.0 is better than 1.0 for VIGS in S. pimpinellifolium, at least in the context of our experiment. Thus, the evaluation method based on PDS is applicable for optimizing the VIGS system in plants.

\section{Silencing efficiency of SpMPK1 at $O D_{600}$ of 2.0}

The results described above show that when the infiltrated concentration was an $\mathrm{OD}_{600}$ of $2.0,92.3 \%$ of the infiltrated plants were VIGS and that leaves 4-9 were optimal samples for further experiments. To validate the accuracy of these results, we attempted to silence the SpMPK1 gene in S. pimpinellifolium. pTRV2:SpMPK1 Agrobacterium $\left(\mathrm{OD}_{600}\right.$ of 2.0) was infiltrated into cotyledons and the silencing efficiency of SpMPK1 was assessed by quantitative RT-PCR at $25 \mathrm{dpi}$. The results showed that 27 VIGS plants were obtained from 30 of the infiltrated plants $(90 \%)$. Of these, 25 plants were effectively silenced, as shown by a significant reduction in SpMPK1 gene transcription levels (50-93\%) in leaves 4-9 (Figure 4). These results further demonstrate that the evaluation method is applicable for studying the function of other genes by improving the overall accuracy of the results.

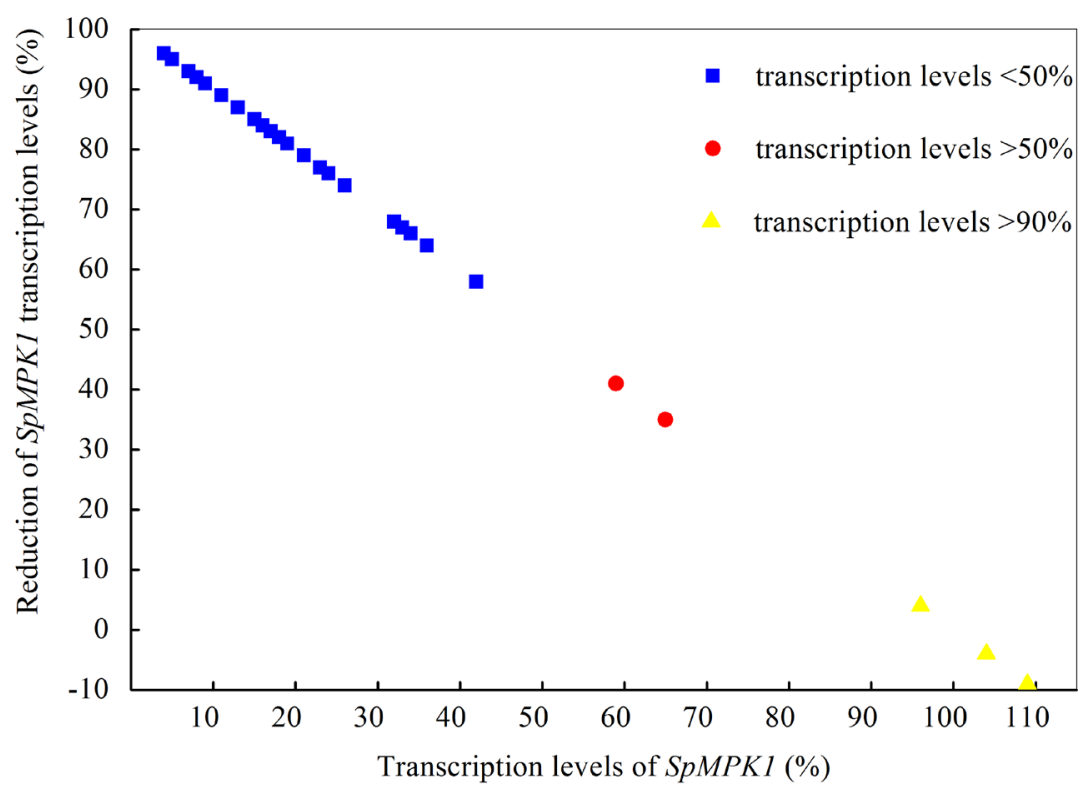

Figure 4. SpMPK1 gene silencing efficiency in leaves 4-9 from VIGS plants. The transcription levels of SpMPK1 were analyzed by quantitative RT-PCR at 25 dpi and presented as the percentages of the mean levels of control plants, which were defined as $100 \%$. 


\section{DISCUSSION}

The TRV-based VIGS vector is one of the most widely used virus vectors and has been repeatedly shown to be useful for studying gene function in plants. However, although many factors related to TRV-induced gene silencing efficiency have been studied, silencing is never $100 \%$ efficient in the tomato plant, nor in any other plant. Therefore, we developed a novel evaluation method to increase the silencing efficiency of candidates used for further experiments.

Typically, $P D S$ is selected as a marker gene in VIGS system optimization, as a reduction in PDS gene transcripts represents the silencing efficiency of VIGS (Rotenberg et al., 2006). For some plant species in which the $P D S$ gene sequences are unknown, the reduction of their transcript abundances within silenced plants is substituted with chlorophyll (SenthilKumar et al., 2007). There is currently no systematic approach for evaluating VIGS efficiency. In this study, our evaluation method included 4 indexes (SFP, SEP, SFL, and SEG). SFP and SEP were used to optimize the VIGS system in other plants. Furthermore, SFL was used to assess the gene silencing position. Lastly, SEG was used to determine that standardized samplings from gene-silenced plant were sufficient for analyzing experimental results when the gene of interest in VIGS presented without phenotypic changes. In addition, the simplicity of identifying the ratio of bleaching areas to quantify gene silencing efficiency is advantageous compared to the more difficult experimental procedures often used, such as identifying gene transcription levels and chlorophyll content.

In conclusion, because VIGS is currently applied in a growing number of plant species and is widely used in functional genomic studies of plants, it is necessary to improve the applicability of VIGS vectors. In this study, we developed a novel evaluation method and demonstrated the applicability of this model for optimizing the VIGS system in other plants as well as in selecting highly silenced candidates for further experiment when genes requiring silencing are present without phenotypic changes.

\section{ACKNOWLEDGMENTS}

We thank the Asian Vegetable Research and Development Center (AVRDC), the World Vegetable Center for supplying the experimental material. Research supported by the "13115" Technology Innovation Project of Shaanxi Province (\#2009ZDKG-14) and the Science and Technology Coordination and Innovation Project of Shaanxi Province (\#2011KTCL02-03).

\section{REFERENCES}

Ahn JW, Kim M, Lim JH, Kim GT, et al. (2004). Phytocalpain controls the proliferation and differentiation fates of cells in plant organ development. Plant J. 38: 969-981.

Bartel DP (2004). MicroRNAs: genomics, biogenesis, mechanism, and function. Cell 116: 281-297.

Baulcombe DC (1999). Fast forward genetics based on virus-induced gene silencing. Curr. Opin. Plant Biol. 2: 109-113.

Benedito VA, Visser PB, Angenent GC and Krens FA (2004). The potential of virus-induced gene silencing for speeding up functional characterization of plant genes. Genet. Mol. Res. 3: 323-341.

Brigneti G, Martín-Hernández AM, Jin H, Chen J, et al. (2004). Virus-induced gene silencing in Solanum species. Plant J. 39: 264-272.

Burch-Smith TM, Anderson JC, Martin GB and Dinesh-Kumar SP (2004). Applications and advantages of virus-induced gene silencing for gene function studies in plants. Plant J. 39: 734-746.

Chen JC, Jiang CZ, Gookin TE, Hunter DA, et al. (2004). Chalcone synthase as a reporter in virus-induced gene silencing 
studies of flower senescence. Plant Mol. Biol. 55: 521-530.

Chung E, Seong E, Kim YC, Chung EJ, et al. (2004). A method of high frequency virus-induced gene silencing in chili pepper (Capsicum annuum L. cv. Bukang). Mol. Cells 17: 377-380.

del Pozo O, Pedley KF and Martin GB (2004). MAPKKKalpha is a positive regulator of cell death associated with both plant immunity and disease. EMBO J. 23: 3072-3082.

Ekengren SK, Liu Y, Schiff M, Dinesh-Kumar SP, et al. (2003). Two MAPK cascades, NPR1, and TGA transcription factors play a role in Pto-mediated disease resistance in tomato. Plant J. 36: 905-917.

Hosseini Tafreshi SA, Shariati M, Mofid MR, Khayam NM, et al. (2012). Heterologous virus-induced gene silencing as a promising approach in plant functional genomics. Mol. Biol. Rep. 39: 2169-2178.

Jin H, Axtell MJ, Dahlbeck D, Ekwenna O, et al. (2002). NPK1, an MEKK1-like mitogen-activated protein kinase kinase kinase, regulates innate immunity and development in plants. Dev. Cell 3: 291-297.

Kandoth PK, Ranf S, Pancholi SS, Jayanty S, et al. (2007). Tomato MAPKs LeMPK1, LeMPK2, and LeMPK3 function in the systemin-mediated defense response against herbivorous insects. Proc. Natl. Acad. Sci. U. S. A. 104: 12205-12210.

Liu H, Fu D, Zhu B, Yan H, et al. (2012). Virus-induced gene silencing in eggplant (Solanum melongena). J. Integr. Plant Biol. 54: 422-429.

Liu Y, Schiff M and Dinesh-Kumar SP (2002a). Virus-induced gene silencing in tomato. Plant J. 31: 777-786.

Liu Y, Schiff M, Marathe R and Dinesh-Kumar SP (2002b). Tobacco Rar1, EDS1 and NPR1/NIM1 like genes are required for N-mediated resistance to tobacco mosaic virus. Plant J. 30: 415-429.

Peart JR, Cook G, Feys BJ, Parker JE, et al. (2002a). An EDS1 orthologue is required for N-mediated resistance against tobacco mosaic virus. Plant J. 29: 569-579.

Peart JR, Lu R, Sadanandom A, Malcuit I, et al. (2002b). Ubiquitin ligase-associated protein SGT1 is required for host and nonhost disease resistance in plants. Proc. Natl. Acad. Sci. U. S. A. 99: 10865-10869.

Ratcliff F, Martin-Hernandez AM and Baulcombe DC (2001). Technical Advance. Tobacco rattle virus as a vector for analysis of gene function by silencing. Plant J. 25: 237-245.

Rotenberg D, Thompson TS, German TL and Willis DK (2006). Methods for effective real-time RT-PCR analysis of virus-induced gene silencing. J. Virol. Methods 138: 49-59.

Ryu CM, Anand A, Kang L and Mysore KS (2004). Agrodrench: a novel and effective agroinoculation method for virusinduced gene silencing in roots and diverse Solanaceous species. Plant J. 40: 322-331.

Saedler R and Baldwin IT (2004). Virus-induced gene silencing of jasmonate-induced direct defences, nicotine and trypsin proteinase-inhibitors in Nicotiana attenuata. J. Exp. Bot. 55: 151-157.

Senthil-Kumar M, Hema R, Anand A, Kang L, et al. (2007). A systematic study to determine the extent of gene silencing in Nicotiana benthamiana and other Solanaceae species when heterologous gene sequences are used for virus-induced gene silencing. New Phytol. 176: 782-791.

Valentine T, Shaw J, Blok VC, Phillips MS, et al. (2004). Efficient virus-induced gene silencing in roots using a modified tobacco rattle virus vector. Plant Physiol. 136: 3999-4009.

van der Knaap E, Sanyal A, Jackson SA and Tanksley SD (2004). High-resolution fine mapping and fluorescence in situ hybridization analysis of sun, a locus controlling tomato fruit shape, reveals a region of the tomato genome prone to DNA rearrangements. Genetics 168: 2127-2140.

Velásquez AC, Chakravarthy S and Martin GB (2009). Virus-induced gene silencing (VIGS) in Nicotiana benthamiana and tomato. J. Vis. Exp. pii: 1292. Doi: 10.3791/1292.

Wu C, Jia L and Goggin F (2011). The reliability of virus-induced gene silencing experiments using tobacco rattle virus in tomato is influenced by the size of the vector control. Mol. Plant Pathol. 12: 299-305. 